\title{
VON NEUMANN REGULARITY OF $V$-RINGS WITH ARTINIAN PRIMITIVE FACTOR RINGS
}

\author{
GIUSEPPE BACCELLA
}

(Communicated by Donald S. Passman)

\begin{abstract}
Let $R$ be a ring whose right primitive factor rings are artinian. It is a known result that if $R$ is von Neumann regular, then $R$ is a right $V$-ring, that is, all simple right $R$-modules are injective. In the present work we prove that the converse holds.
\end{abstract}

All rings we consider are associative with identity and all modules are unital. A ring $R$ is a right $V$-ring if every simple right $R$-module is injective. A well-known result of Kaplansky states that a commutative ring $R$ is (von Neumann) regular if and only if $R$ is a $V$-ring. This is no longer true if one drops the commutativity condition: if $V_{D}$ is an infinite-dimensional vector space over a division ring $D$, then $R=\operatorname{End}\left(V_{D}\right)$ is regular but ${ }_{R} V$ is a simple noninjective module (see [8, Proposition 2.4 ] for a proof), hence $R$ is not a left $V$-ring. On the other hand, there exist simple and noetherian $V$-rings which are not artinian, and hence are not regular (see [3]). A problem which naturally arises is to find classes of rings, containing properly the class of commutative rings, in which the two conditions of being regular and $V$-ring are equivalent. B. Sarath and K. Varadarajan proved in [8, Theorem 3.4] that the above equivalence holds for the class of rings in which all maximal right ideals are two-sided (see also [2, Theorem 3.7]). J. M. Goursaud and J. Valette proved in [6, Theorem 2.3] that if $R$ has all primitive factor rings artinian, then $R$ regular implies that $R$ is a $V$-ring. The purpose of the present paper is to prove the converse: a right $V$-ring all of whose right primitive factor rings are artinian is regular.

A ring $R$ is called fully right idempotent if $I=I^{2}$ for every right ideal $I$ or, equivalently, if ${ }_{R}(R / K)$ is flat for every two-sided ideal $K . R$ is a right $\Sigma$-V-ring if every simple right $R$-module is $\Sigma$-injective. We recall that a right $V$-ring is fully right idempotent (see [7, Corollary 2.2]) and a prime fully right idempotent ring is right nonsingular (see [2, Lemma 4.3]). We also recall that if $K$ is a two-sided ideal of the ring $R$, then ${ }_{R}(R / K)$ is flat if and only if every injective right $R / K$-module is injective as an $R$-module (see [1, Proposition 5]). We shall denote with $r_{R}(M)$ the right annihilator of a right $R$-module $M$.

THEOREM. Let $R$ be a ring all of whose right primitive factor rings are artinian. Then the following conditions are equivalent:

(1) $R$ is a right $V$-ring.

(2) $R$ is a right $\Sigma$ - $V$-ring.

(3) $R$ is fully right idempotent.

(4) $R$ is von Neumann regular.

Received by the editors January 26, 1987 and, in revised form, May 15, 1987. 1980 Mathematics Subject Classification (1985 Revision). Primary 16A30. 
ProOF. (1) $\Rightarrow(3)$. See [7, Corollary 2.2].

$(3) \Rightarrow(2)$. Assume (3), let $S_{R}$ be simple and set $P=r_{R}(S)$.Then ${ }_{R}(R / P)$ is flat and, since $S_{R / P}$ is $\Sigma$-injective, it follows that $S_{R}$ is $\Sigma$-injective.

$(2) \Rightarrow(1)$ is obvious.

$(4) \Rightarrow(3)$ is clear.

$(1) \Rightarrow(4)$. Assume (1). Let us consider first the case in which $R$ is prime and let us prove that, consequently, $R$ is finite (Goldie) dimensional on the right. Since $R$ is right nonsingular, then the maximal right ring of quotients $Q$ of $R$ is von Neumann regular. In order to prove our claim it is sufficient to show that $Q$ is semisimple. Assume, on the contrary, that $Q$ is not semisimple. Then there is an independent sequence $A_{1}, \ldots, A_{n}, \ldots$ of nonzero right ideals of $Q$. Since $R$ is essential in $Q_{R}$, this yields an independent sequence $x_{1} R, \ldots, x_{n} R, \ldots$ of nonzero principal right ideals of $R$, and $x_{1} Q, \ldots, x_{n} Q, \ldots$ is still independent. Inasmuch as $R$ is prime, there are $y_{1}, \ldots, y_{n}, \cdots \in R$ such that $x_{n} y_{n-1} x_{n-1} \cdots \cdot y_{1} x_{1} \neq 0$ for all $n$. For each $n$, let us consider the element $z_{n}=x_{n} y_{n-1} x_{n-1} \cdots \cdot y_{1} x_{1}$ of $R$. We get a descending chain $Q z_{1} \geq \cdots \geq Q z_{n} \geq \cdots$ of nonzero principal left ideals of $Q$. For each $n$ there exists an idempotent $f_{n} \in Q$ such that $Q z_{n}=Q f_{n}$ and we have then an ascending chain $\left(1-f_{1}\right) Q \leq \cdots \leq\left(1-f_{n}\right) Q \leq \cdots$ of proper right ideals of $Q$. Since $R$ is a right $V$-ring, there exists a maximal submodule $M$ of $Q_{R}$ such that $\bigcup_{n}\left(1-f_{n}\right) Q \subset M$ and $1 \notin M$. If $P=r_{R}(Q / M)$, then $P$ is a right primitive ideal of $R$. Since $R$ is fully right idempotent, then ${ }_{R}(R / P)$ is flat and therefore $P=R \cap Q P$; moreover $Q P \subset M$.

We claim that $z_{n} \notin P$ for all $n$. For if $z_{n} \in P$ for some $n$, then $f_{n} \in Q P \subset M$ (since $f_{n}=q_{n} z_{n}$ for some $q_{n} \in Q$ ) and hence $1 \in M$ : a contradiction. Consequently

$$
x_{n} \notin P \text { for all } n \text {. }
$$

Inasmuch as $Q_{Q}$ is injective, it follows from [5, Lemma 9.7] that there are orthogonal idempotents $e_{1}, \ldots, e_{n}, \ldots \in Q$ such that $e_{n} Q=x_{n} Q$ for all $n$. If some $e_{n} \in Q P$, then $x_{n} R=e_{n} x_{n} R \subset Q P \cap R=P$ and hence $x_{n} \in P$, in contradiction with $(*)$. This shows that $\bar{e}_{1} R, \ldots, \bar{e}_{n} R, \ldots$ are nonzero submodules of $(Q / Q P)_{R}$. We claim that they are also independent. Indeed, if there are positive integers $i, j$ with $i<j$ and $\bar{e}_{j} r=\bar{e}_{1} r_{1}+\cdots+\bar{e}_{i} r_{i}$ for some $r, r_{1}, \ldots, r_{i} \in R$, then there is $s \in Q P$ such that $e_{j} r=e_{1} r_{1}+\cdots+e_{i} r_{i}+s$. Since the $e_{n}$ are orthogonal, this yields $e_{j} r=e_{j} e_{j} r=e_{j} s \in Q P$, that is, $\bar{e}_{j} r=0$.

Now $R / P$ is canonically a submodule of $(Q / Q P)_{R}$ and, since each $x_{n} \notin P=$ $R \cap Q P$, the above argument shows that $\bar{x}_{1} R, \ldots, \bar{x}_{n} R, \ldots$ is an infinite sequence of nonzero independent submodules of $(R / P)_{R}$. This is impossible because $R / P$ is artinian. We conclude that $Q$ is semisimple and hence $R_{R}$ is finite-dimensional. Finally it follows from [7, Lemma 3.1] that $R$ is simple; therefore $R$ is artinian by the hypothesis of our theorem.

If $R$ is not prime and $P$ is any prime ideal of $R$, then $R / P$ is a prime right $V$-ring whose right primitive factor rings are artinian; thus $R / P$ is artinian by the above. We see now that $R$ satisfies the hypothesis of [4, Corollary 1.3] and hence $R$ is von Neumann regular. 


\section{REFERENCES}

1. G. Azumaya, Some properties of $T T F$-classes, Proc. Conf. Orders, Group Rings and Related Topics, Lecture Notes in Math., vol. 353, Springer-Verlag, Berlin and New York, 1973, pp. 72-83.

2. G. Baccella, On flat factor rings and fully right idempotent rings, Ann. Univ. Ferrara 26 (1980), 125-141.

3. J. H. Cozzens, Homological properties of the ring of differential polynomials, Bull. Amer. Math. Soc. 76 (1970), 75-79.

4. J. W. Fisher and R. L. Snider, On the von Neumann regularity of rings with regular prime factor rings, Pacific J. Math. 54 (1974), 135-144.

5. K. R. Goodearl, Von Neumann regular rings, Pitman, London/San Francisco/Melbourne, 1979.

6. J. M. Goursaud and J. Valette, Sur l'enveloppe injective des anneaux de groupes réguliers, Bull. Soc. Math. France 103 (1975), 91-102.

7. G. O. Michler and O. E. Villamayor, On rings whose simple modules are injective, J. Algebra 25 (1973), 185-201.

8. B. Sarath and K. Varadarajan, Injectivity of certain classes of modules, J. Pure Appl. Algebra 5 (1974), 293-305.

Dipartimento di Matematica PuRA ed Applicata, Università dell'AQuila, Via ROMA, 67100 L'AQUILA, ITALY 